\title{
QCD studies in $\mathrm{e}^{+} \mathrm{e}^{-}$annihilation from $30 \mathrm{GeV}$ to $189 \mathrm{GeV}$
}

\section{L3 Collaboration}

M. Acciarri ${ }^{\mathrm{z}}$, P. Achard ${ }^{\mathrm{s}}$, O. Adriani ${ }^{\mathrm{p}}$, M. Aguilar-Benitez ${ }^{\mathrm{y}}$, J. Alcaraz $^{\mathrm{y}}$, G. Alemanni ${ }^{\mathrm{v}}$, J. Allaby ${ }^{\mathrm{q}}$, A. Aloisio ${ }^{\mathrm{ab}}$, M.G. Alviggi ${ }^{\mathrm{ab}}$, G. Ambrosi ${ }^{\mathrm{s}}$, H. Anderhub av , V.P. Andreev f,aj, T. Angelescu ${ }^{1}$, F. Anselmo ${ }^{\mathrm{i}}$, A. Arefiev ${ }^{\text {aa }}$, T. Azemoon ${ }^{\mathrm{c}}, \mathrm{T}$. Aziz ${ }^{\mathrm{j}}$, P. Bagnaia ${ }^{\mathrm{ai}}$, A. Bajo ${ }^{\mathrm{y}}$, L. Baksay $^{\mathrm{aq}}, \mathrm{A}$. Balandras ${ }^{\mathrm{d}}$, S.V. Baldew ${ }^{b}$, S. Banerjee ${ }^{j}$, Sw. Banerjee ${ }^{j}$, A. Barczyk ${ }^{\text {av,at }}$, R. Barillère ${ }^{q}$, L. Barone $^{\text {ai }}$, P. Bartalini ${ }^{\mathrm{v}}$, M. Basile ${ }^{\mathrm{i}}$, R. Battiston ${ }^{\text {af }}$, A. Bay ${ }^{\mathrm{v}}$, F. Becattini ${ }^{\mathrm{p}}$, U. Becker ${ }^{\mathrm{n}}$, F. Behner ${ }^{\text {av }}$, L. Bellucci ${ }^{\mathrm{p}}$, R. Berbeco ${ }^{\mathrm{c}}$, J. Berdugo ${ }^{\mathrm{y}}$, P. Berges ${ }^{\mathrm{n}}$, B. Bertucci ${ }^{\text {af }}$, B.L. Betev ${ }^{\text {av }}$, S. Bhattacharya ${ }^{j}$, M. Biasini ${ }^{\text {af }}$, A. Biland ${ }^{\text {av }}$, J.J. Blaising ${ }^{\text {d }}$, S.C. Blyth ${ }^{\text {ag }}$, G.J. Bobbink ${ }^{\mathrm{b}}$, A. Böhm ${ }^{\mathrm{a}}$, L. Boldizsar ${ }^{\mathrm{m}}$, B. Borgia ${ }^{\text {ai }}$, D. Bourilkov ${ }^{\text {av }}$, M. Bourquin ${ }^{\text {s }}$, S. Braccini ${ }^{\text {s }}$, J.G. Branson ${ }^{\text {am }}$, V. Brigljevic ${ }^{\text {av }}$, F. Brochu ${ }^{d}$, A. Buffini ${ }^{p}$, A. Buijs ${ }^{\text {ar }}$, J.D. Burger ${ }^{n}$, W.J. Burger ${ }^{\text {af }}$, X.D. Cai ${ }^{n}$, M. Campanelli av , M. Capell ${ }^{\mathrm{n}}$, G. Cara Romeo ${ }^{\mathrm{i}}$, G. Carlino ${ }^{\mathrm{ab}}$, A.M. Cartacci ${ }^{\mathrm{p}}$, J. Casaus ${ }^{\mathrm{y}}$, G. Castellini ${ }^{\mathrm{p}}$, F. Cavallari ${ }^{\text {ai }}$, N. Cavallo ${ }^{\text {ak }}$, C. Cecchi ${ }^{\text {af }}$, M. Cerrada ${ }^{\mathrm{y}}$, F. Cesaroni ${ }^{\text {w }}$, M. Chamizo ${ }^{\text {s }}$, Y.H. Chang ${ }^{\text {ax }}$, U.K. Chaturvedi ${ }^{r}$, M. Chemarin ${ }^{\mathrm{x}}$, A. Chen ${ }^{\text {ax }}$, G. Chen ${ }^{\mathrm{g}}$, G.M. Chen ${ }^{\mathrm{g}}$, H.F. Chen ${ }^{\mathrm{t}}$, H.S. Chen ${ }^{\mathrm{g}}$, G. Chiefari ${ }^{\mathrm{ab}}$, L. Cifarelli ${ }^{\text {al }}$, F. Cindolo ${ }^{\mathrm{i}}$, C. Civinini ${ }^{\mathrm{p}}$, I. Clare $^{\mathrm{n}}$, R. Clare $^{\mathrm{n}}$, G. Coignet ${ }^{\mathrm{d}}$, N. Colino ${ }^{\mathrm{y}}$, S. Costantini ${ }^{\mathrm{e}}$, F. Cotorobai ${ }^{1}$, B. de la Cruz ${ }^{\mathrm{y}}$, A. Csilling ${ }^{\mathrm{m}}$, S. Cucciarelli af , T.S. Dai ${ }^{\text {n}}$, J.A. van Dalen ${ }^{\text {ad }}$, R. D'Alessandro ${ }^{\text {p }}$, R. de Asmundis ${ }^{\text {ab }}$, P. Déglon ${ }^{\mathrm{s}}$, A. Degré ${ }^{\mathrm{d}}$, K. Deiters ${ }^{\text {at }}$, D. della Volpe ${ }^{\mathrm{ab}}$, E. Delmeire ${ }^{\mathrm{s}}$, P. Denes ${ }^{\text {ah }}$, F. DeNotaristefani ${ }^{\text {ai }}$, A. De Salvo ${ }^{\text {av }}$, M. Diemoz ${ }^{\text {ai }}$, M. Dierckxsens ${ }^{\text {b }}$, D. van Dierendonck ${ }^{\mathrm{b}}$, F. Di Lodovico ${ }^{\text {av }}$, C. Dionisi ${ }^{\text {ai }}$, M. Dittmar ${ }^{\text {av }}$, A. Dominguez $^{\text {am }}$, A. Doria ${ }^{\text {ab }}$, M.T. Dova ${ }^{\mathrm{r}, 5}$, D. Duchesneau ${ }^{\mathrm{d}}$, D. Dufournaud ${ }^{\mathrm{d}}$, P. Duinker ${ }^{\text {b }}$, I. Duran ${ }^{\text {an }}$, H. El Mamouni ${ }^{\mathrm{x}}$, A. Engler ${ }^{\mathrm{ag}}$, F.J. Eppling ${ }^{\text {n }}$, F.C. Erné ${ }^{\mathrm{b}}$, P. Extermann ${ }^{\text {s }}$, M. Fabre ${ }^{\text {at }}$, R. Faccini ${ }^{\text {ai }}$, M.A. Falagan ${ }^{\text {y }}$, S. Falciano ${ }^{\text {ai, }}{ }^{\text {, }}$, A. Favara ${ }^{\text {q }}$, J. Fay ${ }^{\mathrm{x}}$, O. Fedin ${ }^{\text {aj }}$, M. Felcini ${ }^{\text {av }}$, T. Ferguson ${ }^{\text {ag }}$, F. Ferroni ${ }^{\text {ai }}$, H. Fesefeldt ${ }^{\text {a }}$, E. Fiandrini $^{\text {af }}$, J.H. Field ${ }^{\text {s }}$, F. Filthaut ${ }^{\mathrm{q}}$, P.H. Fisher ${ }^{\mathrm{n}}$, I. Fisk ${ }^{\text {am }}$, G. Forconi ${ }^{\mathrm{n}}$, K. Freudenreich ${ }^{\text {av }}$, C. Furetta ${ }^{z}$, Yu. Galaktionov ${ }^{\text {aa, }}$, S.N. Ganguli ${ }^{j}$, P. Garcia-Abia ${ }^{\mathrm{e}}$, M. Gataullin ${ }^{\text {ae }}$, S.S. Gau ${ }^{\text {k }}$, S. Gentile ${ }^{\text {ai, }}$, N. Gheordanescu ${ }^{1}$, S. Giagu ${ }^{\text {ai }}$, Z.F. Gong ${ }^{\text {', }}$ 
G. Grenier ${ }^{\mathrm{x}}$, O. Grimm ${ }^{\text {av }}$, M.W. Gruenewald ${ }^{\mathrm{h}}$, M. Guida ${ }^{\mathrm{al}}$, R. van Gulik ${ }^{\mathrm{b}}$, V.K. Gupta ${ }^{\text {ah }}$, A. Gurtu ${ }^{\text {j, L.J. Gutay }}{ }^{\text {as }}$, D. Haas ${ }^{\text {e }}$, A. Hasan ${ }^{\text {ac }}$, D. Hatzifotiadou ${ }^{\text {i, }}$ T. Hebbeker ${ }^{\text {h}}$, A. Hervé ${ }^{q}$, P. Hidas ${ }^{\mathrm{m}}$, J. Hirschfelder ${ }^{\text {ag }}$, H. Hofer ${ }^{\text {av }}$, G. Holzner ${ }^{\text {av }}$, H. Hoorani ${ }^{\text {ag }}$, S.R. Hou ${ }^{\text {ax }}$, Y. Hu ${ }^{\text {ad }}$, I. Iashvili au, B.N. Jin ${ }^{g}$, L.W. Jones ${ }^{c}$, P. de Jong ${ }^{\text {b }}$, I. Josa-Mutuberría ${ }^{\mathrm{y}}$, R.A. Khan $^{\mathrm{r}}$, M. Kaur ${ }^{\mathrm{r}, 6}$, M.N. Kienzle-Focacci ${ }^{\mathrm{s}}$, D. Kim ${ }^{\text {ai }}$, J.K. Kim ${ }^{\text {ap }}$, J. Kirkby ${ }^{\text {q }}$, D. Kiss ${ }^{\mathrm{m}}$, W. Kittel ${ }^{\text {ad }}$, A. Klimentov ${ }^{\text {n,aa }}$, A.C. König ad ${ }^{\text {ad }}$ A. Kopp ${ }^{\text {au }}$, V. Koutsenko ${ }^{\text {n,aa }}$, M. Kräber ${ }^{\text {av }}$, R.W. Kraemer ${ }^{\text {ag }}$, W. Krenz ${ }^{\text {a }}$, A. Krüger ${ }^{\text {au }}$, A. Kunin ${ }^{\text {naa }}$, P. Ladron de Guevara ${ }^{y}$, I. Laktineh ${ }^{\mathrm{x}}$, G. Landi ${ }^{\mathrm{p}}$, K. Lassila-Perini ${ }^{\text {av }}$, M. Lebeau ${ }^{\mathrm{q}}$, A. Lebedev ${ }^{\mathrm{n}}$, P. Lebrun ${ }^{\mathrm{x}}$, P. Lecomte ${ }^{\text {av }}$, P. Lecoq ${ }^{q}$, P. Le Coultre ${ }^{\text {av }}$, H.J. Lee ${ }^{\text {h }}$, J.M. Le Goff ${ }^{\mathrm{q}}$, R. Leiste ${ }^{\text {au }}$, E. Leonardi ai ${ }^{\text {, }}$ P. Levtchenko ${ }^{\text {aj }}$, C. Li ${ }^{t}$, S. Likhoded ${ }^{\text {au }}$, C.H. Lin ${ }^{\text {ax }}$, W.T. Lin ${ }^{\text {ax }}$, F.L. Linde ${ }^{b}$, L. Lista ${ }^{\mathrm{ab}}$, Z.A. Liu ${ }^{\mathrm{g}}$, W. Lohmann ${ }^{\text {au }}$, E. Longo ${ }^{\text {a }}$, Y.S. Lu ${ }^{\mathrm{g}}$, K. Lübelsmeyer ${ }^{\text {a }}$, C. Luci ${ }^{\text {qai }}$, D. Luckey ${ }^{n}$, L. Lugnier ${ }^{\mathrm{x}}$, L. Luminari ${ }^{\text {ai }}$, W. Lustermann ${ }^{\text {av }}$, W.G. Ma ${ }^{\mathrm{t}}$, M. Maity ${ }^{\mathrm{j}}$, L. Malgeri ${ }^{\mathrm{q}}$, A. Malinin ${ }^{\mathrm{q}}$, C. Maña ${ }^{\mathrm{y}}$, D. Mangeol ${ }^{\text {ad }}$, J. Mans ${ }^{\text {ah }}$, P. Marchesini av , G. Marian o , J.P. Martin ${ }^{\text {x }}$, F. Marzano ai, K. Mazumdar ${ }^{\text {j, }}$, R.R. McNeil ${ }^{\text {}}$, S. Mele ${ }^{\mathrm{q}}$, L. Merola ${ }^{\mathrm{ab}}$, M. Meschini ${ }^{\mathrm{p}}$, W.J. Metzger ${ }^{\text {ad }}$, M. von der Mey ${ }^{\text {a }}$, A. Mihul ${ }^{1}$, H. Milcent ${ }^{\mathrm{q}}$, G. Mirabelli ${ }^{\text {ai }}$, J. Mnich ${ }^{\mathrm{q}}$, G.B. Mohanty ${ }^{\text {j, P. Molnar }}{ }^{\text {, }}$, T. Moulik ${ }^{\text {j }}$, G.S. Muanza ${ }^{x}$, A.J.M. Muijs ${ }^{\text {b }}$, B. Musicar ${ }^{\text {am }}$, M. Musy ${ }^{\text {ai }}$, M. Napolitano ${ }^{\text {ab }}$, F. Nessi-Tedaldi ${ }^{\text {av }}$, H. Newman ${ }^{\text {ae }}$, T. Niessen ${ }^{\text {a }}$, A. Nisati ${ }^{\text {ai }}$, H. Nowak ${ }^{\text {au }}$, G. Organtini ${ }^{\text {ai }}$, A. Oulianov ${ }^{\text {aa }}$, C. Palomares ${ }^{\mathrm{y}}$, D. Pandoulas ${ }^{\mathrm{a}}$, S. Paoletti ${ }^{\text {ai, }}$, P. Paolucci ${ }^{\text {ab }}$, R. Paramatti ${ }^{\text {ai }}$, H.K. Park ${ }^{\text {ag }}$, I.H. Park ${ }^{\text {ap }}$, G. Passaleva ${ }^{\text {q }}$, S. Patricelli ${ }^{\text {ab }}$, T. Paul ${ }^{\text {k }}$, M. Pauluzzi ${ }^{\text {af }}$, C. Paus ${ }^{\text {q }}$, F. Pauss ${ }^{\text {av }}$, M. Pedace ${ }^{\text {ai }}$, S. Pensotti ${ }^{z}$, D. Perret-Gallix ${ }^{\text {d }}$, B. Petersen ${ }^{\text {ad }}$, D. Piccolo ${ }^{\text {ab }}$, F. Pierella ${ }^{i}$, M. Pieri ${ }^{\text {p }}$, P.A. Piroué ${ }^{\text {ah }}$, E. Pistolesi ${ }^{z}$, V. Plyaskin ${ }^{\text {aa }}$, M. Pohl ${ }^{\text {s }}$, V. Pojidaev ${ }^{\text {aa, }}$, H. Postema ${ }^{n}$, J. Pothier ${ }^{\text {q }}$, D.O. Prokofiev ${ }^{\text {as }}$, D. Prokofiev ${ }^{\text {aj }}$, J. Quartieri ${ }^{\text {al }}$, G. Rahal-Callot ${ }^{\text {av,q }}$, M.A. Rahaman ${ }^{j}$, P. Raics ${ }^{\circ}$, N. Raja ${ }^{j}$, R. Ramelli ${ }^{\text {av }}$, P.G. Rancoita ${ }^{z}$, A. Raspereza ${ }^{\text {au }}$, G. Raven ${ }^{\text {am }}$, P. Razis ${ }^{\text {ac }}$, D. Ren ${ }^{\text {av }}$, M. Rescigno ${ }^{\text {ai }}$, S. Reucroft ${ }^{k}$, S. Riemann ${ }^{\text {au }}$, K. Riles ${ }^{\text {c }}$, A. Robohm ${ }^{\text {av }}$, J. Rodin ${ }^{\text {aq }}$, B.P. Roe ${ }^{\text {c }}$, L. Romero ${ }^{\mathrm{y}}$, A. Rosca ${ }^{\mathrm{h}}$, S. Rosier-Lees ${ }^{\mathrm{d}}$, J.A. Rubio ${ }^{\mathrm{q}}$, G. Ruggiero $^{\mathrm{p}}$, D. Ruschmeier ${ }^{\mathrm{h}}$, H. Rykaczewski ${ }^{\text {av }}$, S. Saremi ${ }^{\mathrm{f}}$, S. Sarkar ${ }^{\text {ai }}$, J. Salicio ${ }^{\text {q, E. Sanchez }}{ }^{\text {q }}$, M.P. Sanders ${ }^{\text {ad }}$, M.E. Sarakinos ${ }^{\text {u }}$, C. Schäfer ${ }^{\text {q, }}$, V. Schegelsky ${ }^{\text {aj }}$, S. Schmidt-Kaerst ${ }^{\text {a }}$, D. Schmitz ${ }^{\text {a }}$, H. Schopper ${ }^{\text {aw }}$, D.J. Schotanus ${ }^{\text {ad }}$, G. Schwering ${ }^{\text {a }}$, C. Sciacca ${ }^{\text {ab }}$, D. Sciarrino ${ }^{\text {s }}$, A. Seganti ${ }^{i}$, L. Servoli ${ }^{\text {af }}$, S. Shevchenko ${ }^{\text {ae }}$, N. Shivarov ${ }^{\text {ao }}$, V. Shoutko ${ }^{\text {aa }}$, E. Shumilov ${ }^{\text {aa }}$, A. Shvorob ${ }^{\text {ae }}$, T. Siedenburg ${ }^{\mathrm{a}}$, D. Son ${ }^{\text {ap }}$, B. Smith ${ }^{\mathrm{ag}}$, P. Spillantini ${ }^{\mathrm{p}}$, M. Steuer ${ }^{\mathrm{n}}$, D.P. Stickland ${ }^{\text {ah }}$, A. Stone ${ }^{f}$, B. Stoyanov ${ }^{\text {ao }}$, A. Straessner ${ }^{\text {a }}$, K. Sudhakar ${ }^{\text {j, }}$, G. Sultanov ${ }^{r}$, L.Z. Sun ${ }^{\mathrm{t}}$, H. Suter ${ }^{\text {av }}$, J.D. Swain ${ }^{\mathrm{r}}$, Z. Szillasi ${ }^{\text {aq,3 }}{ }^{\text {, T. Sztaricskai }}{ }^{\text {aq, } 3 \text {, }}$ X.W. Tang ${ }^{g}$, L. Tauscher ${ }^{\mathrm{e}}$, L. Taylor ${ }^{\mathrm{k}}$, B. Tellili ${ }^{\mathrm{x}}$, C. Timmermans ${ }^{\text {ad }}$, 
Samuel C.C. Ting ${ }^{\text {n }}$, S.M. Ting ${ }^{\text {n }}$, S.C. Tonwar ${ }^{\mathrm{j}}$, J. Tóth ${ }^{\mathrm{m}}$, C. Tully ${ }^{\mathrm{q}}$, K.L. Tung ${ }^{\mathrm{g}}$, Y. Uchida ${ }^{\mathrm{n}}$, J. Ulbricht ${ }^{\text {av }}$, E. Valente ${ }^{\text {ai }}$, G. Vesztergombi ${ }^{\mathrm{m}}$, I. Vetlitsky ${ }^{\text {aa }}$, D. Vicinanza ${ }^{\text {al }}$, G. Viertel ${ }^{\text {av }}$, S. Villa ${ }^{\mathrm{k}}$, M. Vivargent ${ }^{\mathrm{d}}$, S. Vlachos ${ }^{\mathrm{e}}$, I. Vodopianov ${ }^{\text {aj }}$, H. Vogel ${ }^{\text {ag }}$, H. Vogt ${ }^{\text {au }}$, I. Vorobiev ${ }^{\text {aa }}$, A.A. Vorobyov ${ }^{\text {aj }}$, A. Vorvolakos ${ }^{\text {ac }}$, M. Wadhwa ${ }^{\mathrm{e}}$, W. Wallraff ${ }^{\mathrm{a}}$, M. Wang ${ }^{\mathrm{n}}$, X.L. Wang ${ }^{\mathrm{t}}$, Z.M. Wang ${ }^{\mathrm{t}}$, A. Weber ${ }^{\text {a }}$, M. Weber ${ }^{\text {a }}$, P. Wienemann ${ }^{\text {a }}$, H. Wilkens ${ }^{\text {ad }}$, S.X. Wu ${ }^{\text {n }}$, S. Wynhoff ${ }^{\mathrm{q}}$, L. Xia ${ }^{\text {ae }}$, Z.Z. Xu ${ }^{\text {t }}$, J. Yamamoto ${ }^{\text {c }}$, B.Z. Yang ${ }^{\text {t }}$, C.G. Yang ${ }^{g}$, H.J. Yang ${ }^{g}$, M. Yang ${ }^{g}$, J.B. Ye ${ }^{\mathrm{t}}$, S.C. Yeh ${ }^{\text {ay }}$, An. Zalite ${ }^{\text {aj }}$, Yu. Zalite ${ }^{\text {aj }}$, Z.P. Zhang ${ }^{\mathrm{t}}$, G.Y. Zhu ${ }^{\mathrm{g}}$, R.Y. Zhu ${ }^{\text {ae }}$, A. Zichichi ${ }^{\text {i,q,r }}$, G. Zilizi aq,3 ${ }^{\text {a }}$, M. Zöller ${ }^{\text {a }}$

a I. Physikalisches Institut, RWTH, D-52056 Aachen, Germany, and III. Physikalisches Institut, RWTH, D-52056 Aachen, Germany ${ }^{1}$

${ }^{\mathrm{b}}$ National Institute for High Energy Physics, NIKHEF, and University of Amsterdam, NL-1009 DB Amsterdam, The Netherlands ${ }^{\mathrm{c}}$ University of Michigan, Ann Arbor, MI 48109, USA

${ }^{\mathrm{d}}$ Laboratoire d'Annecy-le-Vieux de Physique des Particules, LAPP, IN2P3-CNRS, BP 110, F-74941 Annecy-le-Vieux CEDEX, France

' Institute of Physics, University of Basel, CH-4056 Basel, Switzerland

${ }^{\mathrm{f}}$ Louisiana State University, Baton Rouge, LA 70803, USA

${ }^{\mathrm{g}}$ Institute of High Energy Physics, IHEP, 100039 Beijing, China ${ }^{7}$

${ }^{\mathrm{h}}$ Humboldt University, D-10099 Berlin, Germany ${ }^{1}$

${ }^{\mathrm{i}}$ University of Bologna and INFN-Sezione di Bologna, I-40126 Bologna, Italy

${ }^{\mathrm{j}}$ Tata Institute of Fundamental Research, Bombay 400 005, India

${ }^{\mathrm{k}}$ Northeastern University, Boston, MA 02115, USA

${ }^{1}$ Institute of Atomic Physics and University of Bucharest, R-76900 Bucharest, Romania

${ }^{m}$ Central Research Institute for Physics of the Hungarian Academy of Sciences, H-1525 Budapest 114, Hungary ${ }^{2}$

${ }^{\mathrm{n}}$ Massachusetts Institute of Technology, Cambridge, MA 02139, USA

${ }^{\circ}$ KLTE-ATOMKI, H-4010 Debrecen, Hungary ${ }^{3}$

${ }^{\mathrm{p}}$ INFN Sezione di Firenze and University of Florence, I-50125 Florence, Italy

${ }^{\mathrm{q}}$ European Laboratory for Particle Physics, CERN, CH-1211 Geneva 23, Switzerland

${ }^{\mathrm{r}}$ World Laboratory, FBLJA Project, CH-1211 Geneva 23, Switzerland

${ }^{\mathrm{s}}$ University of Geneva, CH-1211 Geneva 4, Switzerland

${ }^{\mathrm{t}}$ Chinese University of Science and Technology, USTC, Hefei, Anhui 230 029, China ${ }^{7}$

u SEFT, Research Institute for High Energy Physics, P.O. Box 9, SF-00014 Helsinki, Finland

${ }^{v}$ University of Lausanne, CH-1015 Lausanne, Switzerland

${ }^{\mathrm{w}}$ INFN-Sezione di Lecce and Universitá Degli Studi di Lecce, I-73100 Lecce, Italy

${ }^{\mathrm{x}}$ Institut de Physique Nucléaire de Lyon, IN2P3-CNRS, Université Claude Bernard, F-69622 Villeurbanne, France

${ }^{\mathrm{y}}$ Centro de Investigaciones Energéticas, Medioambientales y Tecnologícas, CIEMAT, E-28040 Madrid, Spain ${ }^{4}$

${ }^{\mathrm{z}}$ INFN-Sezione di Milano, I-20133 Milan, Italy

aa Institute of Theoretical and Experimental Physics, ITEP, Moscow, Russia

${ }^{\mathrm{ab}}$ INFN-Sezione di Napoli and University of Naples, I-80125 Naples, Italy

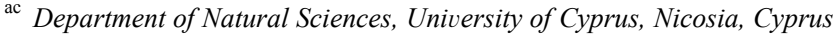

${ }^{\text {ad }}$ University of Nijmegen and NIKHEF, NL-6525 ED Nijmegen, The Netherlands

${ }^{\text {ae }}$ California Institute of Technology, Pasadena, CA 91125, USA

af INFN-Sezione di Perugia and Universitá Degli Studi di Perugia, I-06100 Perugia, Italy

${ }^{\text {ag }}$ Carnegie Mellon University, Pittsburgh, PA 15213, USA

ah Princeton University, Princeton, NJ 08544, USA

ai INFN-Sezione di Roma and University of Rome, 'La Sapienza', I-00185 Rome, Italy

${ }^{\text {aj }}$ Nuclear Physics Institute, St. Petersburg, Russia

ak INFN-Sezione di Napoli and University of Potenza, I-85100 Potenza, Italy

${ }^{\text {al }}$ University and INFN, Salerno, I-84100 Salerno, Italy

am University of California, San Diego, CA 92093, USA

${ }^{a n}$ Dept. de Fisica de Particulas Elementales, Univ. de Santiago, E-15706 Santiago de Compostela, Spain

ao Bulgarian Academy of Sciences, Central Lab. of Mechatronics and Instrumentation, BU-1113 Sofia, Bulgaria

ap Laboratory of High Energy Physics, Kyungpook National University, 702-701 Taegu, South Korea 


\author{
${ }^{\text {aq }}$ University of Alabama, Tuscaloosa, AL 35486, USA \\ ${ }^{\text {ar }}$ Utrecht University and NIKHEF, NL-3584 CB Utrecht, The Netherlands \\ as Purdue University, West Lafayette, IN 47907, USA \\ ${ }^{\text {at }}$ Paul Scherrer Institut, PSI, CH-5232 Villigen, Switzerland \\ au DESY, D-15738 Zeuthen, Germany \\ av Eidgenössische Technische Hochschule, ETH Zürich, CH-8093 Zürich, Switzerland \\ ${ }^{\text {aw }}$ University of Hamburg, D-22761 Hamburg, Germany \\ ${ }^{a x}$ National Central University, Chung-Li, Taiwan, ROC \\ ${ }^{\text {ay }}$ Department of Physics, National Tsing Hua University, Taiwan, ROC
}

Received 5 May 2000; accepted 2 August 2000

Editor: K. Winter

\begin{abstract}
We present results obtained from a study of the structure of hadronic events recorded by the L3 detector at various centre-of-mass energies. The distributions of event shape variables and the energy dependence of their mean values are measured from $30 \mathrm{GeV}$ to $189 \mathrm{GeV}$ and compared with various QCD models. The energy dependence of the moments of event shape variables is used to test a power law ansatz for the non-perturbative component. We obtain a universal value of the non-perturbative parameter $\alpha_{0}=0.537 \pm 0.073$. From a comparison with resummed $\mathscr{O}\left(\alpha_{\mathrm{s}}^{2}\right)$ QCD calculations, we determine the strong coupling constant at each of the selected energies. The measurements demonstrate the running of $\alpha_{\mathrm{s}}$ as expected in QCD with a value of $\alpha_{\mathrm{s}}\left(m_{\mathrm{Z}}\right)=0.1215 \pm 0.0012$ (exp) \pm 0.0061 (th). (C) 2000 Elsevier Science B.V. All rights reserved.
\end{abstract}

\section{Introduction}

LEP operated at centre-of-mass energies around 91.2 GeV from 1989 to 1995 and then moved up to six different centre-of-mass energies between 130 $\mathrm{GeV}$ and $189 \mathrm{GeV}$ in the following three years. Thus a study of the process $\mathrm{e}^{+} \mathrm{e}^{-} \rightarrow$ hadrons at LEP offers a unique environment to test the predic-

\footnotetext{
E-mail address: salvatore.mele@cern.ch (S. Mele).

${ }^{1}$ Supported by the German Bundesministerium für Bildung, Wissenschaft, Forschung und Technologie.

${ }^{2}$ Supported by the Hungarian OTKA fund under contract numbers T019181, F023259 and T024011.

${ }^{3}$ Also supported by the Hungarian OTKA fund under contract numbers T22238 and T026178.

${ }^{4}$ Supported also by the Comisión Interministerial de Ciencia y Tecnología.

${ }^{5}$ Also supported by CONICET and Universidad Nacional de La Plata, CC 67, 1900 La Plata, Argentina.

${ }^{6}$ Also supported by Panjab University, Chandigarh-160014, India.

${ }^{7}$ Supported by the National Natural Science Foundation of China.
}

tions of the theory of the strong interaction (QCD) over a wide energy range. The energy range has been extended by using hadronic events from $\mathrm{Z}$ decays with isolated high energy photons in order to probe the structure of hadronic events at reduced centreof-mass energies down to $30 \mathrm{GeV}$ [1,2]. The high energy photons are radiated early in the process through initial state radiation (ISR) or through quark bremsstrahlung whereas the hadronic shower develops over a longer time scale.

We report here measurements of event shape distributions and their moments using the data collected with the L3 detector [3]. We update the published results at $\sqrt{s}=161,172$ and $183 \mathrm{GeV}[4,5]$ with an improved selection method for hadronic events and present new results at $\sqrt{s}=130,136$ and $189 \mathrm{GeV}$. The measured distributions are compared with predictions from event generators based on an improved leading log approximation (Parton Shower models including QCD coherence effects). Three such Monte Carlo programs (JETSET PS [6], Herwig [7] and ARIADNE [8]) have been used for these comparisons. We also compare our measure- 
ments with predictions from QCD models with no coherence effects (COJETs [9]). These Monte Carlo programs use different approaches to describe both the perturbative parton shower evolution and nonperturbative hadronisation processes. They have been tuned to reproduce the global event shape distributions and the charged particle multiplicity distribution measured at $91.2 \mathrm{GeV}[10]$.

The moments of event shape variables are measured between $30 \mathrm{GeV}$ and $189 \mathrm{GeV}$. The perturbative and non-perturbative QCD contributions are obtained from a fit using the power correction formula [11]. This approach was first applied by the DELPHI collaboration [12].

The strong coupling constant $\alpha_{\mathrm{s}}$ is also determined at each of these centre-of-mass energies by comparing the measured event shape distributions with predictions of second order QCD calculations [13] containing resummed leading and next-to-leading order terms [14].

Section 2 describes the selection of hadronic events. Measurements of event shape variables and estimation of systematic errors are described in Section 3. Section 4 presents a comparison of the data with predictions from various QCD models, a study of the power correction ansatz and a determination of $\alpha_{\mathrm{s}}$ from event shape distributions. The results are summarised in Section 5.

\section{Event selection}

The selection of $\mathrm{e}^{+} \mathrm{e}^{-} \rightarrow$ hadrons events is based on the energy measured in the electromagnetic and hadron calorimeters. We use energy clusters in the calorimeters with a minimum energy of 100 $\mathrm{MeV}$. We measure the total visible energy $\left(E_{\mathrm{vis}}\right)$ and the energy imbalances parallel $\left(E_{\|}\right)$and perpendicular $\left(E_{\perp}\right)$ to the beam direction. Backgrounds are different for hadronic $\mathrm{Z}$ decays, hadronic events at reduced centre-of-mass energies and at high energies. This is reflected in the different selection cuts used for these three types of data sets.

We use Monte Carlo events to estimate the efficiency of the selection criteria and purity of the data sample. Monte Carlo events for the process $\mathrm{e}^{+} \mathrm{e}^{-} \rightarrow$ $\mathrm{q} \overline{\mathrm{q}}(\gamma)$ have been generated by the parton shower programs JETSET and PYTHIA [15] and passed through the L3 detector simulation [16]. The background events are simulated with appropriate event generators: PythiA and PHOJET [17] for two-photon events, Koralz [18] for the $\tau^{+} \tau^{-}(\gamma)$ final state, Bhagene [19] and BHWIDE [20] for Bhabha events, KORALW [21] for W-pair production and PYTHIA for Z-pair production.

Details of event selection at $\sqrt{s} \approx m_{\mathrm{Z}}$ and at reduced centre-of-mass energies have been described earlier [1,2]. At $\sqrt{s} \approx m_{\mathrm{Z}}$, we have used only a small subset of the complete data sample $\left(8.3 \mathrm{pb}^{-1}\right.$ out of $142.4 \mathrm{pb}^{-1}$ of integrated luminosity) which still provides an experimental error three times smaller than theoretical uncertainties.

Data at $\sqrt{s}=130$ and $136 \mathrm{GeV}$ were collected in two separate runs during 1995 [4] and 1997. The main background at these energies comes from ISR resulting in a mass of the hadronic system close to $m_{\mathrm{Z}}$. This background is reduced by applying a cut in the two dimensional plane of $\left|E_{\|}\right| / E_{\mathrm{vis}}$ and $E_{\text {vis }} / \sqrt{s}$. In the current analysis, data sets from the two years have been combined and the cuts are optimised to get the best efficiency times purity.

For the data at $\sqrt{s} \geq 161 \mathrm{GeV}$, additional backgrounds arise from $\mathrm{W}$-pair and Z-pair production. A

Table 1

Summary of integrated luminosity, selection efficiency, sample purity and number of selected hadronic events at the different energies used in this analysis. The energies below $\sqrt{s}=91 \mathrm{GeV}$ are obtained from the full data sample at the $\mathrm{Z}$ peak, by selecting events with an isolated high energy photon

\begin{tabular}{crrrr}
\hline$\sqrt{s}$ & $\begin{array}{r}\text { Integrated } \\
\text { luminosity } \\
\left(\mathrm{pb}^{-1}\right)\end{array}$ & $\begin{array}{r}\text { Selection } \\
\text { efficiency } \\
(\%)\end{array}$ & $\begin{array}{r}\text { Sample } \\
\text { purity } \\
(\%)\end{array}$ & $\begin{array}{r}\text { Selected } \\
\text { events }\end{array}$ \\
\hline $30-50$ & 142.4 & 48.3 & 68.4 & 1247 \\
$50-60$ & 142.4 & 41.0 & 78.0 & 1047 \\
$60-70$ & 142.4 & 35.2 & 86.0 & 1575 \\
$70-80$ & 142.4 & 29.9 & 89.0 & 2938 \\
$80-84$ & 142.4 & 27.4 & 90.5 & 2091 \\
$84-86$ & 142.4 & 27.5 & 87.0 & 1607 \\
91.2 & 8.3 & 98.5 & 99.8 & 248100 \\
130 & 6.1 & 90.0 & 80.6 & 556 \\
136 & 5.9 & 89.0 & 81.5 & 414 \\
161 & 10.8 & 89.0 & 81.2 & 424 \\
172 & 10.2 & 84.8 & 82.6 & 325 \\
183 & 55.3 & 84.2 & 82.4 & 1500 \\
189 & 176.8 & 87.8 & 81.1 & 4479 \\
\hline
\end{tabular}


substantial fraction $(\sim 80 \%)$ of these events can be removed by a specific selection [5] based on:

- forcing the event to a 4-jet topology using the Durham algorithm [22],

- performing a kinematic fit imposing the constraints of energy-momentum conservation,

- making cuts on energies of the most and the least energetic jets and on $y_{34}^{\mathrm{D}}$, where $y_{34}^{\mathrm{D}}$ is the jet resolution parameter for which the event is classified as a three-jet rather than a four-jet event.

These cuts have also been optimised at each energy point. For centre-of-mass energies at or above 130
$\mathrm{GeV}$, hadronic events with ISR photon energy larger than $0.18 \sqrt{s}$ are considered as background.

The integrated luminosity, selection efficiency, purity and number of selected events for each of the energy points are summarised in Table 1.

\section{Measurement of event shape variables}

We measure five global event shape variables for which improved analytical QCD calculations [14] are available. These are thrust $(T)$ [23], scaled heavy jet
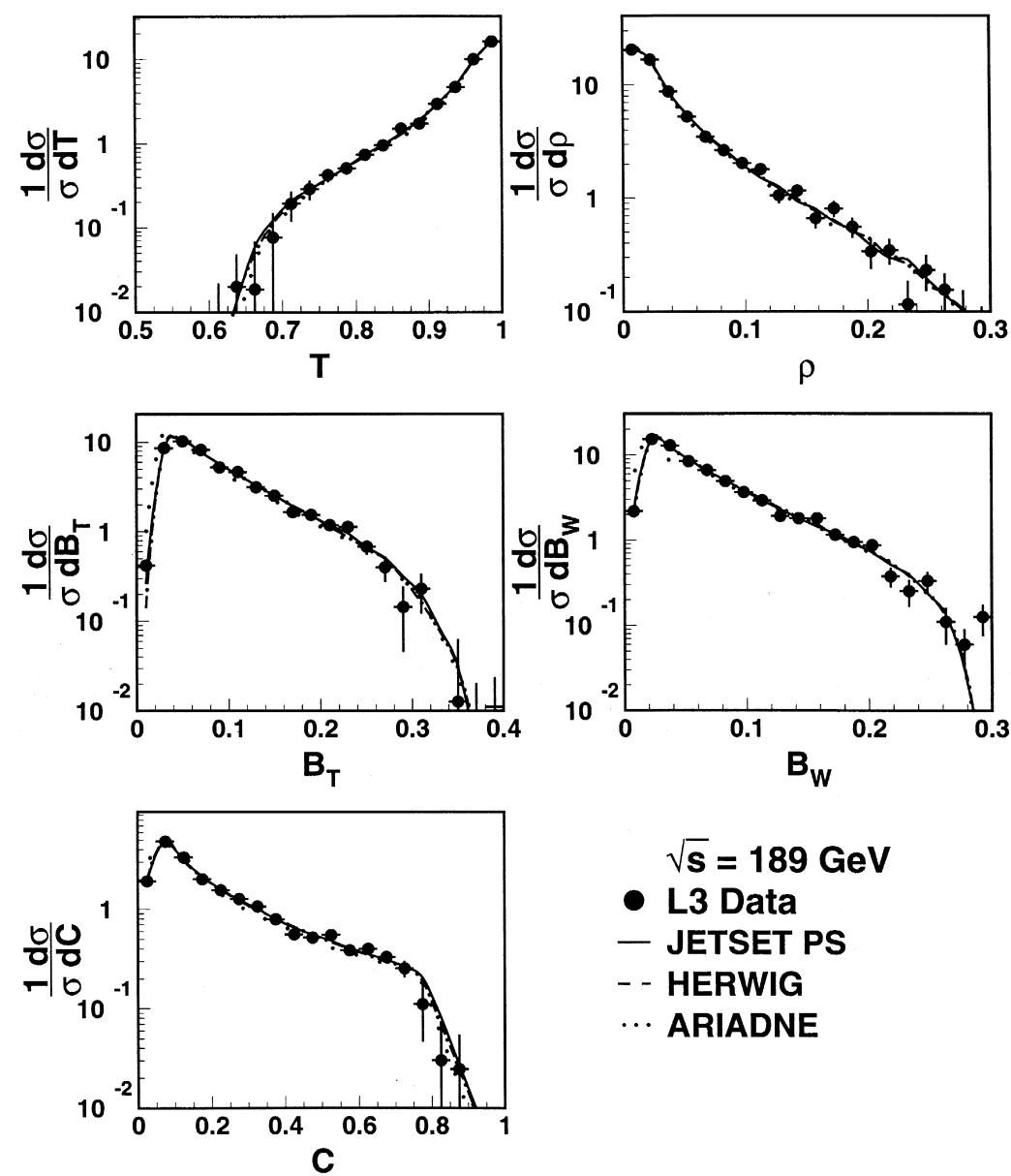

Fig. 1. Distributions for thrust, $T$, scaled heavy jet mass, $\rho$, total and wide jet broadenings, $B_{T}$ and $B_{W}$, and the $C$-parameter at $\sqrt{s}=189$ $\mathrm{GeV}$ in comparison with QCD model predictions. The errors shown are statistical only. 
mass $(\rho)$ [24], total $\left(B_{T}\right)$ and wide $\left(B_{W}\right)$ jet broadening variables [14] and the $C$-parameter [25].

For Monte Carlo events, the global event shape variables are calculated before (particle level) and after (detector level) detector simulation. The calculation before detector simulation takes into account all stable charged and neutral particles. The measured distributions at detector level differ from the ones at particle level because of detector effects, limited acceptance and resolution. After subtracting the background obtained from simulations, the measured distributions for all energies except $\sqrt{s} \approx m_{\mathrm{Z}}$ are corrected for detector effects, acceptance and resolution on a bin-by-bin basis by comparing the detector level results with the particle level results. The level of migration is kept at a negligible level with a bin size larger than the experimental resolution. At $\sqrt{s} \approx m_{\mathrm{Z}}$, the detector effects are unfolded for these event shape variables using a regularised unfolding method [26]. We also correct the data for initial and final state photon radiation bin-by-bin using Monte Carlo distributions at particle level with and without radiation.

The systematic uncertainties in the distributions of event shape variables arise mainly due to uncertainties in the estimation of detector correction and
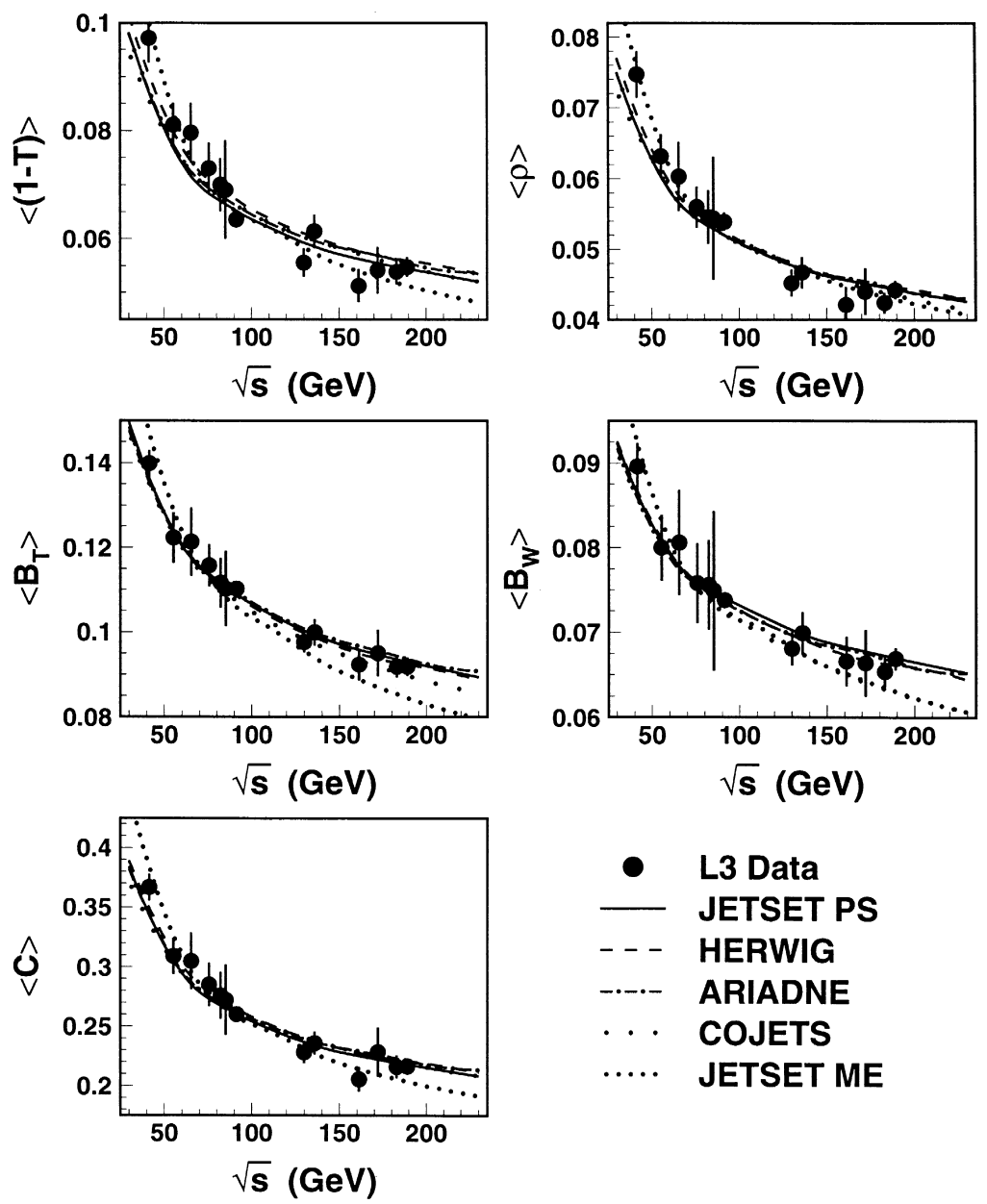

Fig. 2. The first moments of the five event shape variables, $1-T, \rho, B_{T}, B_{W}$ and $C$, as a function of the centre-of-mass energy, compared with several QCD models. 
background estimation. The uncertainty in the detector correction has been estimated by several independent checks:

- The definition of reconstructed objects used to calculate the observables has been changed. Instead of using only calorimetric clusters, the analysis has been repeated with objects obtained from a non-linear combination of energies of charged tracks and calorimetric clusters. At $\sqrt{s} \approx m_{\mathrm{Z}}$, we use a track based selection and the event shape variables are constructed from the tracks.

- The effect of different particle densities in correcting the measured distribution has been estimated by changing the signal Monte Carlo program (HERWIG instead of JETSET).

- The acceptance has been reduced by restricting the events to the central part of the detector $\left(\left|\cos \left(\theta_{\mathrm{T}}\right)\right|<0.7\right.$, where $\theta_{\mathrm{T}}$ is the polar angle of the thrust axis relative to the beam direction) where the energy resolution is better.

The uncertainty on the background composition of the selected event sample has been estimated differently for the three types of data sets. At $\sqrt{s} \approx$ $m_{\mathrm{Z}}$, the background contamination is negligible and the uncertainty due to that has been neglected. For data samples at reduced centre-of-mass energies, the systematic errors arising from background subtraction have been estimated [2] by:

- varying, by one standard deviation, the background scale factor which takes into account the lack of isolated $\pi^{0}$ and $\eta$ production in the Monte Carlo sample,

- varying the cuts on neural network probability, jet and local isolation angles, and energy in the local isolation cone.

At high energies, the uncertainty is determined by repeating the analysis with:

- an alternative criterion to reject the hard initial state photon events based on a cut on the kinematically reconstructed effective centre-of-mass energy,

- a variation of the estimated two-photon interaction background by $\pm 30 \%$ and by changing the background Monte Carlo program (PHOJET instead of PyTHIA), and

- a variation of the $\mathrm{W}^{+} \mathrm{W}^{-}$background estimate by changing the $\mathrm{W}$-pair rejection criteria.

The systematic uncertainties obtained from different sources are combined in quadrature. At high energies, uncertainties due to ISR and $\mathrm{W}^{+} \mathrm{W}^{-}$back-

Table 2

First moments of the five event shape variables at different energy points. The two errors are respectively statistical and systematic

\begin{tabular}{|c|c|c|c|c|c|}
\hline \multirow{2}{*}{$\begin{array}{c}\sqrt{s} \\
(\mathrm{GeV})\end{array}$} & \multicolumn{5}{|c|}{ First moments of } \\
\hline & $1-T$ & $\rho$ & $B_{T}$ & $B_{W}$ & $C$ \\
\hline $30-50$ & $.0971 \pm .0030 \pm .0034$ & $747 \pm .0023 \pm .0023$ & $.1399 \pm .0027 \pm .0016$ & $.0896 \pm .0021 \pm .0018$ & $.3667 \pm .0084 \pm .0073$ \\
\hline $50-60$ & $.0811 \pm .0027 \pm .0029$ & $632 \pm .0021 \pm .0023$ & $.1223 \pm .0025 \pm .0054$ & $.0800 \pm .0020 \pm .0034$ & $.3091 \pm .0080 \pm .0131$ \\
\hline $60-70$ & $.0796 \pm .0021 \pm .0051$ & $.0603 \pm .0015 \pm .0047$ & $.1213 \pm .0019 \pm .0079$ & $.0806 \pm .0014 \pm .0060$ & $.3049 \pm .0059 \pm .0232$ \\
\hline $70-80$ & $.0731 \pm .0015 \pm .0045$ & $.0560 \pm .0011 \pm .0027$ & $.1157 \pm .0015 \pm .0048$ & $.0758 \pm .0011 \pm .0046$ & $.2851 \pm .0044 \pm .0177$ \\
\hline $80-84$ & $.0700 \pm .0018 \pm .0046$ & $.0546 \pm .0015 \pm .0035$ & $.1116 \pm .0017 \pm .00$ & $.0756 \pm .0014 \pm .0051$ & $.2759 \pm .00$ \\
\hline $84-86$ & $.0691 \pm .0022 \pm .0088$ & $.0544 \pm .0017 \pm .0085$ & $.1102 \pm .0021 \pm .0086$ & $.0749 \pm .0017 \pm .0092$ & $.2722 \pm .0068 \pm$. \\
\hline 91.2 & $.0636 \pm .0003 \pm .0013$ & $.0539 \pm .0002 \pm .0013$ & $.1102 \pm .0002 \pm .0011$ & $.0738 \pm .0001 \pm .0008$ & $.2599 \pm .0004 \pm$ \\
\hline 130 & $.0556 \pm .0022 \pm .0014$ & $.0452 \pm .0018 \pm .0007$ & $.0976 \pm .0023 \pm .0008$ & $.0681 \pm .0019 \pm .0007$ & $.2277 \pm .0072 \pm .0052$ \\
\hline 136 & $.0614 \pm .0029 \pm .0011$ & $.0467 \pm .0022 \pm .0004$ & $.0999 \pm .0029 \pm .0011$ & $.0699 \pm .0024 \pm .0006$ & $.2357 \pm .0089 \pm .0038$ \\
\hline 161 & $.0513 \pm .0030 \pm .0008$ & $.0421 \pm .0025 \pm .0007$ & $.0923 \pm .0032 \pm .0018$ & $.0666 \pm .0027 \pm .0010$ & $.2052 \pm .0098 \pm .0028$ \\
\hline 172 & $.0542 \pm .0037 \pm .0022$ & $.0440 \pm .0028 \pm .0018$ & $.0950 \pm .0046 \pm .0031$ & $.0664 \pm .0031 \pm .0023$ & $.2281 \pm .0159 \pm .0133$ \\
\hline 183 & $.0539 \pm .0020 \pm .0011$ & $.0424 \pm .0014 \pm .0004$ & $.0918 \pm .0020 \pm .0015$ & $.0654 \pm .0015 \pm .0010$ & $.2157 \pm .0063 \pm .0073$ \\
\hline 189 & $.0548 \pm .0013 \pm .0013$ & $.0442 \pm .0009 \pm .0009$ & $.0918 \pm .0013 \pm .0018$ & $.0669 \pm .0009 \pm .0010$ & $.2160 \pm .0040 \pm .0041$ \\
\hline
\end{tabular}


Table 3

Determination of $\alpha_{0}$ and $\alpha_{\mathrm{s}}\left(m_{\mathrm{Z}}\right)$ from fits to the first moments of the event shape distributions together with $\chi^{2} /$ d.o.f. from those fits. Also shown in the last two columns are the $A_{2}$ parameter from fits to the second moments and the resulting $\chi^{2} /$ d.o.f.

\begin{tabular}{lllrrr}
\hline Observable & $\alpha_{0}$ & $\alpha_{\mathrm{s}}\left(m_{\mathrm{Z}}\right)$ & $\chi^{2} /$ d.o.f. & \multicolumn{1}{c}{$A_{2}\left(\mathrm{GeV}^{2}\right)$} & $\chi^{2} /$ d.o.f. \\
\hline $1-T$ & $0.633 \pm 0.097$ & $0.1104 \pm 0.0065$ & $11.5 / 11$ & $4.54 \pm 0.99$ & $10.3 / 12$ \\
$\rho$ & $0.523 \pm 0.063$ & $0.1027 \pm 0.0050$ & $5.5 / 11$ & $0.69 \pm 0.63$ & $3.4 / 12$ \\
$B_{T}$ & $0.517 \pm 0.044$ & $0.1160 \pm 0.0029$ & $3.5 / 11$ & $9.78 \pm 1.74$ & $21.9 / 12$ \\
$B_{W}$ & $0.476 \pm 0.100$ & $0.1134 \pm 0.0042$ & $4.1 / 11$ & $-5.53 \pm 1.95$ & $17.0 / 12$ \\
$C$ & $0.537 \pm 0.044$ & $0.1125 \pm 0.0038$ & $6.3 / 11$ & $7.28 \pm 1.81$ & $13.1 / 12$ \\
\hline
\end{tabular}

grounds are the most important ones. They are roughly equal and are 2-3 times larger than the uncertainties due to the detector correction.
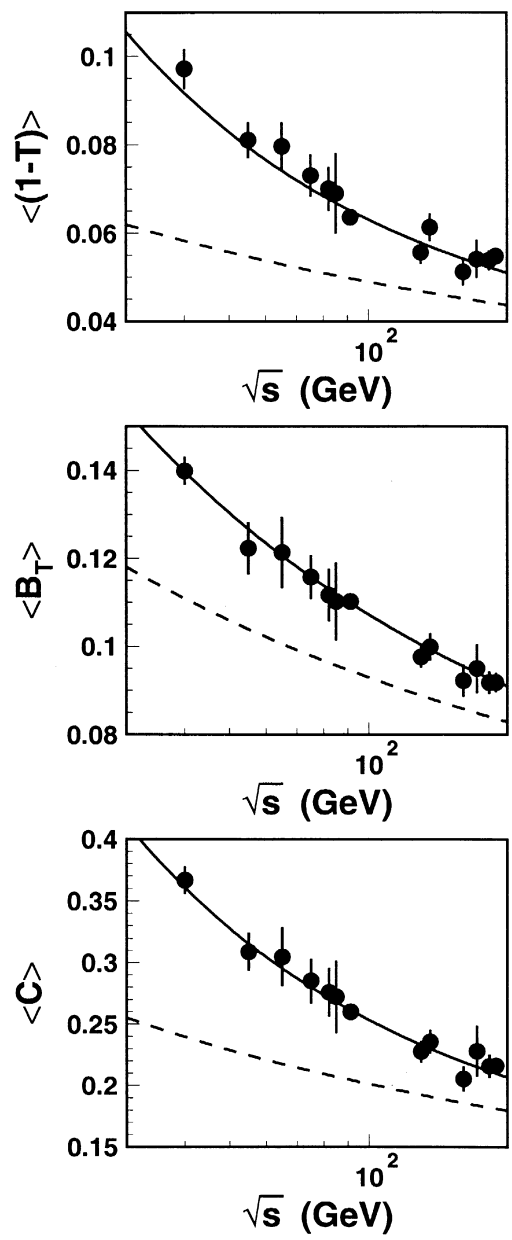

Apart from the data set at $\sqrt{s} \approx m_{\mathrm{Z}}$, statistical fluctuations are not negligible in the estimation of systematic effects. The statistical component of the
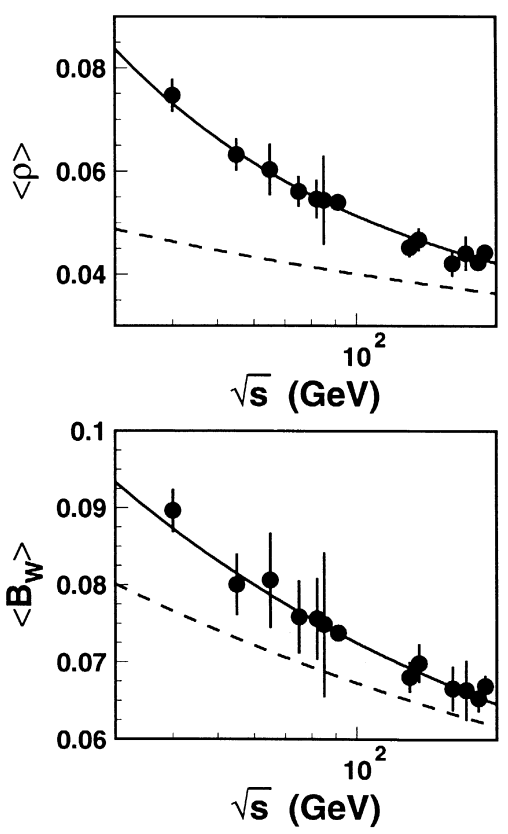

- L3 Data

- Fit

- - - Perturbative component

Fig. 3. The first moments of the five event shape variables, $1-T, \rho, B_{T}, B_{W}$ and $C$ compared to the results of a fit including perturbative and power law contributions. 
Table 4

Second moments of the five event shape variables at different energy points. The two errors are respectively statistical and systematic

\begin{tabular}{|c|c|c|c|c|c|}
\hline $\begin{array}{c}\sqrt{s} \\
(\mathrm{GeV})\end{array}$ & \multicolumn{5}{|c|}{ Second moments of } \\
\hline $30-50$ & $.0143 \pm .0009 \pm .0015$ & $.0080 \pm .0006 \pm .0005$ & $.0236 \pm .0009 \pm .0005$ & $.0104 \pm .0005 \pm .0005$ & $.1726 \pm .0078 \pm .0115$ \\
\hline $60-70$ & $.0109 \pm .0006 \pm .0010$ & $.0060 \pm .0004 \pm .0011$ & $.0187 \pm .0006 \pm .0022$ & $.0088 \pm .0003 \pm .0013$ & $.1308 \pm .0050 \pm .0164$ \\
\hline $70-80$ & $.0093 \pm .0004 \pm .0010$ & $.0053 \pm .0002 \pm .0007$ & $.0172 \pm .0005 \pm .0014$ & $.0081 \pm .0003 \pm .0008$ & $.1176 \pm .0037 \pm .0117$ \\
\hline $80-84$ & $.0086 \pm .0005 \pm .0010$ & $.0052 \pm .0003 \pm .0007$ & $.0160 \pm .0006 \pm .0015$ & $.0081 \pm .0003 \pm .0008$ & $.1110 \pm .0047 \pm .0125$ \\
\hline 91.2 & $.0077 \pm .0001 \pm .0003$ & $.0053 \pm .0001 \pm .0002$ & $.0158 \pm .0001 \pm .0003$ & $.0076 \pm .0001 \pm .0002$ & $.1034 \pm .0003 \pm .0031$ \\
\hline 130 & $.0064 \pm .0005 \pm .0002$ & $.0041 \pm .0003 \pm .0001$ & $.0131 \pm .0006 \pm .0002$ & $.0069 \pm .0004 \pm .0001$ & $.0848 \pm .0050 \pm .0025$ \\
\hline 136 & $.0080 \pm .0008 \pm .0007$ & $.0045 \pm .0004 \pm .0001$ & $.0141 \pm .0008 \pm .0004$ & $.0076 \pm .0005 \pm .0002$ & $.0938 \pm .0064 \pm .0017$ \\
\hline 161 & $.0059 \pm .0007 \pm .0002$ & $.0040 \pm .0004 \pm .0001$ & $.0121 \pm .0008 \pm .0004$ & $.0070 \pm .0005 \pm .0002$ & $.0757 \pm .0064 \pm .0019$ \\
\hline 172 & $.0064 \pm .0009 \pm .0005$ & $.0040 \pm .0005 \pm .0003$ & $.0136 \pm .0014 \pm .0013$ & $.0068 \pm .0006 \pm .0005$ & $.0979 \pm .0133 \pm .0129$ \\
\hline 183 & $.0064 \pm .0005 \pm .0001$ & $.0042 \pm .0003 \pm .0002$ & $.0121 \pm .0006 \pm .0003$ & $.0067 \pm .0003 \pm .0002$ & $.0804 \pm .0051 \pm .0032$ \\
\hline
\end{tabular}

systematic uncertainty is determined by splitting the overall Monte Carlo sample into luminosity weighted sub-samples and treating each of these sub-samples as data. The spread in the mean position gives an estimate of the statistical component and is taken out from the original estimate in quadrature.

\section{Results}

\subsection{Comparison with $Q C D$ models}

Fig. 1 shows the corrected distributions for thrust, scaled heavy jet mass, total and wide jet broadening and the $C$-parameter obtained at $\sqrt{s}=189 \mathrm{GeV}$. The data are compared with predictions from QCD models JETSET PS, Herwig and ARIADNE at particle level. The agreement is satisfactory.

An important test of QCD models is a comparison of the energy evolution of the event shape variables. The energy dependence of the mean event shape variables arises mainly from two sources: the logarithmic energy scale dependence of $\alpha_{\mathrm{s}}$ and the power law behaviour of non-perturbative effects. The first moments of the five event shape variables are shown in Fig. 2 and Table 2. Also shown are the energy dependences of these quantities as predicted by JETset PS, Herwig, Ariadne, Cojets and Jetset ME $\left(\mathscr{O}\left(\alpha_{\mathrm{s}}^{2}\right)\right.$ matrix element implementation). All the models with the possible exception of JETSET ME give a good description of the data.

\subsection{Power law correction analysis}

The energy dependence of moments of the event shape variables has been described [11] as a sum of the perturbative contributions and a power law dependence due to non-perturbative contributions. The first moment of an event shape variable $f$ is written as

$$
\langle f\rangle=\left\langle f_{\text {pert }}\right\rangle+\left\langle f_{\text {pow }}\right\rangle,
$$

where the perturbative contribution $\left\langle f_{\text {pert }}\right\rangle$ has been determined to $\mathscr{O}\left(\alpha_{\mathrm{s}}^{2}\right)$ [27]. The power correction term [11], for $1-T, \rho$, and $C$, is given by

$$
\left\langle f_{\text {pow }}\right\rangle=c_{f} \mathscr{P},
$$

where the factor $c_{f}$ depends on the shape variable $f$ and $\mathscr{P}$ is supposed to have a universal form:

$$
\begin{aligned}
\mathscr{P}= & \frac{4 C_{F}}{\pi^{2}} \mathscr{M} \frac{\mu_{I}}{\sqrt{s}}\left[\alpha_{0}\left(\mu_{I}\right)-\alpha_{\mathrm{s}}(\sqrt{s})\right. \\
& \left.-\beta_{0} \frac{\alpha_{\mathrm{s}}^{2}(\sqrt{s})}{2 \pi}\left(\ln \frac{\sqrt{s}}{\mu_{I}}+\frac{K}{\beta_{0}}+1\right)\right]
\end{aligned}
$$



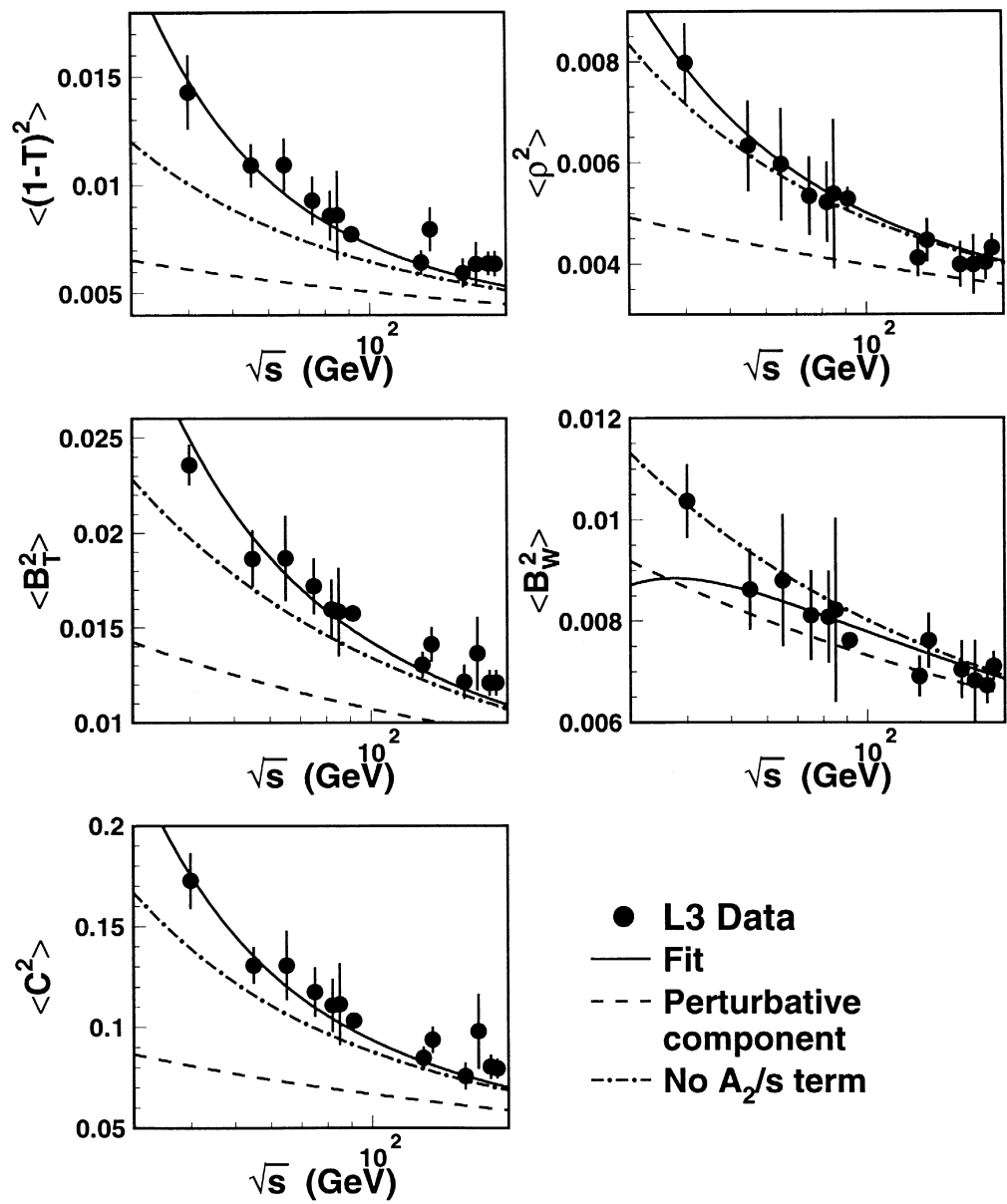

Fig. 4. The second moments of the five event shape variables, $1-T, \rho, B_{T}, B_{W}$ and $C$ compared to the results of a fit including perturbative and power law contributions. The parameters $\alpha_{0}$ and $\alpha_{\mathrm{s}}$ are fixed to the values obtained by the fits to the first moments. The $A_{2} / s$ term is very small for $\rho$, negative for $B_{W}$ but is positive and necessary to reproduce the behaviour of $1-T, B_{T}$ and $C$.

for a renormalisation scale fixed at $\sqrt{s}$. The parameter $\alpha_{0}$ is related to the value of $\alpha_{\mathrm{s}}$ in the non-perturbative region below an infrared matching scale $\mu_{I}$ $(=2 \mathrm{GeV}) ; \beta_{0}$ is $\left(11 N_{c}-2 N_{f}\right) / 3$, where $N_{c}$ is the number of colours and $N_{f}$ is the number of active flavours. $K=\left(67 / 18-\pi^{2} / 6\right) C_{A}-5 N_{f} / 9$ and $C_{F}, C_{A}$ are the usual colour factors. The Milan factor $\mathscr{M}$ is 1.49 for $N_{f}=3$. For the jet broadening variables, the power correction term takes the form

$$
\left\langle f_{\text {pow }}\right\rangle=c_{f} F \mathscr{P},
$$

where

$$
\begin{aligned}
F= & \left(\frac{\pi}{2 \sqrt{a C_{F} \alpha_{\mathrm{CMW}}}}+\frac{3}{4}-\frac{\beta_{0}}{6 a C_{F}}\right. \\
& \left.-0.6137+\mathscr{O}\left(\sqrt{\alpha_{\mathrm{s}}}\right)\right)
\end{aligned}
$$

and $a$ takes a value 1 for $B_{T}$ and 2 for $B_{W}$ and $\alpha_{\mathrm{CMW}}$ is related to $\alpha_{\mathrm{s}}$ [11].

We have carried out fits to the first moments of the five event shape variables separately with $\alpha_{\mathrm{s}}\left(m_{\mathrm{Z}}\right)$ 
and $\alpha_{0}$ as free parameters. The diagonal terms of the covariance matrix between the different energy points are constructed by summing in quadrature the systematic uncertainty and the statistical error. The offdiagonal terms are obtained from the common systematic errors. The results of the fits are summarised in Table 3 and shown in Fig. 3. The correlation coefficient between $\alpha_{\mathrm{s}}\left(m_{\mathrm{Z}}\right)$ and $\alpha_{0}$ is about -0.9 .

The five values of $\alpha_{0}$ obtained from the event shape variables agree within errors, supporting the predicted universality of the power law behaviour. The theoretical predictions for event shape variables,

Table 5

$\alpha_{\mathrm{s}}$ measured at $\sqrt{s}=130,136,161,172,183$ and $189 \mathrm{GeV}$ from fits of the event shape variables to theoretical predictions with combined fixed order and resummed calculations. The fit ranges, the estimated experimental and theoretical errors and the fit quality are also given

\begin{tabular}{|c|c|c|c|c|c|}
\hline & $(1-T)$ & $\rho$ & $B_{T}$ & $B_{W}$ & $C$ \\
\hline Fit range: & $\overline{0.00-0.30}$ & $\overline{0.00-0.20}$ & $\overline{0.00-0.25}$ & $\overline{0.00-0.20}$ & $0.05-0.50$ \\
\hline$\alpha_{\mathrm{s}}(130 \mathrm{GeV})$ & 0.1139 & 0.1134 & 0.1153 & 0.1063 & 0.1151 \\
\hline statistical error & \pm 0.0036 & \pm 0.0034 & \pm 0.0027 & \pm 0.0027 & \pm 0.0036 \\
\hline systematic error & \pm 0.0028 & \pm 0.0029 & \pm 0.0016 & \pm 0.0015 & \pm 0.0018 \\
\hline overall experimental error & \pm 0.0046 & \pm 0.0045 & \pm 0.0031 & \pm 0.0031 & \pm 0.0040 \\
\hline overall theoretical error & \pm 0.0056 & \pm 0.0038 & \pm 0.0062 & \pm 0.0088 & \pm 0.0066 \\
\hline$\chi^{2} /$ d.o.f. & $6.9 / 10$ & $8.4 / 9$ & $9.1 / 11$ & $12.0 / 12$ & $8.5 / 8$ \\
\hline$\alpha_{\mathrm{s}}(136 \mathrm{GeV})$ & 0.1166 & 0.1112 & 0.1141 & 0.1045 & 0.1089 \\
\hline statistical error & \pm 0.0047 & \pm 0.0037 & \pm 0.0034 & \pm 0.0032 & \pm 0.0043 \\
\hline systematic error & \pm 0.0024 & \pm 0.0013 & \pm 0.0010 & \pm 0.0026 & \pm 0.0020 \\
\hline overall experimental error & \pm 0.0053 & \pm 0.0039 & \pm 0.0035 & \pm 0.0041 & \pm 0.0047 \\
\hline overall theoretical error & \pm 0.0060 & \pm 0.0037 & \pm 0.0064 & \pm 0.0078 & \pm 0.0076 \\
\hline$\chi^{2} /$ d.o.f. & $10.2 / 9$ & $11.4 / 13$ & $7.7 / 11$ & $7.9 / 12$ & $11.8 / 8$ \\
\hline$\alpha_{\mathrm{s}}(161 \mathrm{GeV})$ & 0.1018 & 0.1012 & 0.1101 & 0.1032 & 0.1043 \\
\hline statistical error & \pm 0.0051 & \pm 0.0052 & \pm 0.0039 & \pm 0.0039 & \pm 0.0055 \\
\hline Systematic error & \pm 0.0022 & \pm 0.0022 & \pm 0.0015 & \pm 0.0044 & \pm 0.0025 \\
\hline overall experimental error & \pm 0.0056 & \pm 0.0056 & \pm 0.0042 & \pm 0.0059 & \pm 0.0060 \\
\hline overall theoretical error & \pm 0.0050 & \pm 0.0034 & \pm 0.0066 & \pm 0.0068 & \pm 0.0057 \\
\hline$\chi^{2} /$ d.o.f. & $8.2 / 9$ & $5.7 / 13$ & $\overline{7} .9 / 11$ & $\overline{5} .6 / 12$ & $4.9 / 8$ \\
\hline$\alpha_{\mathrm{s}}(172 \mathrm{GeV})$ & 0.1109 & 0.1099 & 0.1071 & 0.1020 & 0.1121 \\
\hline statistical error & \pm 0.0055 & \pm 0.0050 & \pm 0.0043 & \pm 0.0039 & \pm 0.0064 \\
\hline systematic error & \pm 0.0026 & \pm 0.0016 & \pm 0.0044 & \pm 0.0022 & \pm 0.0024 \\
\hline overall experimental error & \pm 0.0061 & \pm 0.0052 & \pm 0.0062 & \pm 0.0045 & \pm 0.0068 \\
\hline overall theoretical error & \pm 0.0064 & \pm 0.0033 & \pm 0.0060 & \pm 0.0065 & \pm 0.0057 \\
\hline$\chi^{2} /$ d.o.f. & $2.8 / 8$ & $8.4 / 13$ & $\overline{7} .8 / 12$ & $8.4 / 13$ & $3.2 / 8$ \\
\hline$\alpha_{\mathrm{s}}(183 \mathrm{GeV})$ & 0.1132 & 0.1075 & 0.1112 & 0.1036 & 0.1081 \\
\hline statistical error & \pm 0.0023 & \pm 0.0022 & \pm 0.0017 & \pm 0.0015 & \pm 0.0028 \\
\hline systematic error & \pm 0.0012 & \pm 0.0011 & \pm 0.0013 & \pm 0.0006 & \pm 0.0010 \\
\hline overall experimental error & \pm 0.0026 & \pm 0.0025 & \pm 0.0021 & \pm 0.0016 & \pm 0.0029 \\
\hline overall theoretical error & \pm 0.0054 & \pm 0.0038 & \pm 0.0060 & \pm 0.0071 & \pm 0.0054 \\
\hline$\chi^{2} /$ d.o.f. & $4.2 / 11$ & $6.4 / 13$ & $15.9 / 12$ & $6.3 / 13$ & $5.2 / 8$ \\
\hline$\alpha_{\mathrm{s}}(189 \mathrm{GeV})$ & 0.1168 & 0.1108 & 0.1114 & 0.1033 & 0.1118 \\
\hline statistical error & \pm 0.0014 & \pm 0.0013 & \pm 0.0011 & \pm 0.0010 & \pm 0.0018 \\
\hline systematic error & \pm 0.0012 & \pm 0.0010 & \pm 0.0014 & \pm 0.0012 & \pm 0.0014 \\
\hline overall experimental error & \pm 0.0018 & \pm 0.0016 & \pm 0.0018 & \pm 0.0016 & \pm 0.0023 \\
\hline overall theoretical error & \pm 0.0057 & \pm 0.0033 & \pm 0.0067 & \pm 0.0078 & \pm 0.0055 \\
\hline$\chi^{2} /$ d.o.f. & $4.4 / 11$ & $8.2 / 13$ & $28.0 / 12$ & $10.6 / 13$ & $5.7 / 8$ \\
\hline
\end{tabular}


being incomplete, give different estimates of $\alpha_{0}$ and $\alpha_{\mathrm{s}}$. Since the measurements are fully correlated, the best estimates of the overall values are obtained by taking an unweighted average:

$\alpha_{0}=0.537 \pm 0.070 \pm 0.021$,

$\alpha_{\mathrm{s}}\left(m_{\mathrm{Z}}\right)=0.1110 \pm 0.0045 \pm 0.0034$.

The first error on each measurement is experimental and is obtained from the average of the five errors on $\alpha_{0}$ and $\alpha_{\mathrm{s}}$. To estimate theoretical uncertainties we vary the renormalisation scale between $0.5 \sqrt{s}$ and $2.0 \sqrt{s}$ and $\alpha_{0}$ and $\alpha_{\mathrm{s}}\left(m_{\mathrm{Z}}\right)$ vary on average by \pm 0.021 and \pm 0.0033 respectively. A variation of $\mu_{I}$ in the range from 1 to $3 \mathrm{GeV}$ gives an additional uncertainty on $\alpha_{\mathrm{s}}\left(m_{\mathrm{Z}}\right)$ of \pm 0.0010 . These two estimates of theoretical uncertainties are combined in quadrature and quoted as the second error.

We have also measured the second moments of these shape variables which are summarised in Table 4. The energy dependence of these moments has been analysed in terms of power law corrections. For variables $1-T, \rho$ and $C$, the following result is expected to hold [28]:

$$
\left\langle f^{2}\right\rangle=\left\langle f_{\text {pert }}^{2}\right\rangle+2\left\langle f_{\text {pert }}\right\rangle c_{f} \mathscr{P}+\mathscr{O}\left(\frac{1}{s}\right) .
$$

This assumes that the non-perturbative correction to the distributions causes only a shift. For jet broadenings the power corrections are more complicated. The $\mathscr{O}\left(\frac{1}{s}\right)$ term has been parametrised as $A_{2} / s$ and is expected to be small for $1-T, \rho$ and $C$. Fits have been performed to the second moments where $\alpha_{0}$ and $\alpha_{\mathrm{s}}$ have been fixed to the values obtained from the corresponding fits to the first moments. Fig. 4 shows the second moments compared to these fits. The contributions of the $\mathscr{O}\left(\frac{1}{s}\right)$ term are non-negligible for $1-T$ and $C$, in contradiction with the expectation. The fit to $B_{W}$ gives a negative $A_{2}$ value which gives a poor description of the evolution in the low energy range. The five values of $A_{2}$, as obtained from the fits, are summarised in Table 3.

\section{3. $\alpha_{\mathrm{s}}$ from event shape distributions}

In order to derive $\alpha_{\mathrm{s}}$ from event shape variables at each energy point we fit the measured distributions to theoretical calculations based on $\mathscr{O}\left(\alpha_{\mathrm{s}}^{2}\right)$ perturbative QCD with resummed leading and nextto-leading order terms. These calculations are performed at parton level and do not include heavy quark mass effects. To compare the analytical calculations with the experimental distributions, the effects of hadronisation and decays have been corrected for using Monte Carlo programs.

The fit ranges used take into account the limited statistics at high energy as well as the reliability of the resummation calculation and are given in Table 5. In this analysis, we determine $\alpha_{\mathrm{s}}$ at $\sqrt{s}=130$, 136 and $189 \mathrm{GeV}$ for the first time. We also include the measurements done at $\sqrt{s}=161,172$ and 183

Table 6

Summary of $\alpha_{\mathrm{s}}$ values as determined from event shape variables at different centre-of-mass energies. The $\alpha_{\mathrm{s}}$ values for $\sqrt{s} \leq m_{\mathrm{Z}}$ were determined $[1,2]$ only from four event shape variables for which analytical calculations were available at that time

\begin{tabular}{ccc}
\hline$\sqrt{s}(\mathrm{GeV})$ & $\alpha_{\mathrm{s}}\left(\right.$ from $\left.T, \rho, B_{T}, B_{W}\right)$ & $\alpha_{\mathrm{s}}\left(\right.$ from $\left.T, \rho, B_{T}, B_{W}, C\right)$ \\
\hline $30-50$ & $0.1400 \pm 0.0056 \pm 0.0107$ & \\
$50-60$ & $0.1260 \pm 0.0073 \pm 0.0088$ & \\
$60-70$ & $0.1340 \pm 0.0060 \pm 0.0087$ & \\
$70-80$ & $0.1210 \pm 0.0064 \pm 0.0082$ & \\
$80-84$ & $0.1200 \pm 0.0057 \pm 0.0089$ & $0.1128 \pm 0.0038 \pm 0.0063$ \\
$84-86$ & $0.1160 \pm 0.0061 \pm 0.0082$ & $0.111 \pm \pm 0.0043 \pm 0.0061$ \\
91.2 & $0.1221 \pm 0.0020 \pm 0.0066$ & $0.1041 \pm 0.0054 \pm 0.0054$ \\
130 & $0.1122 \pm 0.0038 \pm 0.0060$ & $0.1084 \pm 0.0056 \pm 0.0055$ \\
136 & $0.1116 \pm 0.0042 \pm 0.0060$ & $0.1088 \pm 0.0023 \pm 0.0055$ \\
161 & $0.1041 \pm 0.0052 \pm 0.0054$ & $0.1105 \pm 0.0018 \pm 0.0058$ \\
172 & $0.1075 \pm 0.0054 \pm 0.0056$ & \\
183 & $0.1089 \pm 0.0022 \pm 0.0056$ & \\
\hline
\end{tabular}


$\mathrm{GeV}$ since the experimental systematic uncertainties are considerably reduced by using an improved selection method and by subtracting the statistical component of the systematic uncertainties. All the measurements are summarised in Table 5. These measurements supersede those published previously [5].

The experimental errors include the statistical errors and the experimental systematic uncertainties. The theoretical error is obtained from estimates [5] of the hadronisation uncertainty and of the errors coming from the uncalculated higher orders in the QCD predictions. The estimate of the theoretical error does not always reflect the true size of uncalculated higher order terms. An independent estimate is obtained from a comparison of $\alpha_{\mathrm{s}}$ measurements from many event shape variables which are affected differently by higher order corrections and hadronisation effects. To obtain a combined value for the strong coupling constant we take the unweighted average of the five $\alpha_{\mathrm{s}}$ values. We estimate the overall theoretical error from the simple average of the five theoretical errors or from half of the maximum spread in the five $\alpha_{\mathrm{s}}$ values. Both estimates yield similar results. The combined results are summarised in Table 6 . The earlier measurements at $\sqrt{s}$ $=m_{\mathrm{Z}}$ and at reduced centre-of-mass energies determined $\alpha_{\mathrm{s}}$ from four event shape variables only: $T$, $\rho, B_{T}$ and $B_{W}$. For comparison we also provide in Table 6 the mean from these four measurements.

We compare the energy dependence of the measured $\alpha_{\mathrm{s}}$ values with the prediction from QCD in Fig. 5a. The theoretical errors are strongly correlated between these measurements. The error appropriate to a measurement of the energy dependence of $\alpha_{\mathrm{s}}$ can then be considered to be experimental. The experimental systematic errors on $\alpha_{\mathrm{s}}$ are dominated by the background uncertainties. These are similar for all the individual low energy or high energy data points but differ between the low energy, $\mathrm{Z}$ peak and high energy data sets. The experimental systematic errors are then different and uncorrelated between the three data sets, but are taken as fully correlated between individual low energy or high energy measurements. The thirteen measurements in Fig. 5a are shown with experimental errors only, together with a fit to the QCD evolution equation [29] with $\alpha_{\mathrm{s}}\left(m_{\mathrm{Z}}\right)$ as a free parameter. The fit gives a $\chi^{2}$ of 13.5 for 12
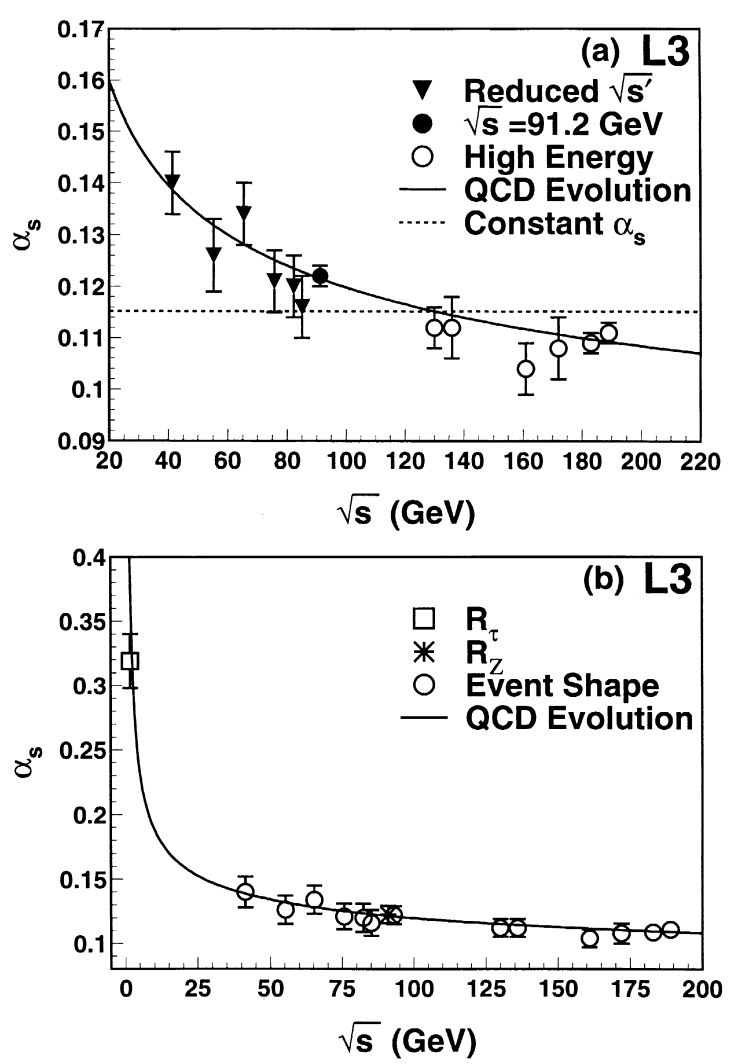

Fig. 5. a) $\alpha_{\mathrm{s}}$ measurements from event shape distributions as a function of the centre-of-mass energy. The errors shown are experimental only. The solid and dashed lines are fits with the energy dependence of $\alpha_{\mathrm{s}}$ as expected from QCD and with constant $\alpha_{\mathrm{s}}$, respectively. b) $\alpha_{\mathrm{s}}$ values as determined by L3 from the $\tau$ lifetime measurement, $\mathrm{Z}$ lineshape and event shape distributions. The line is a fit to the QCD evolution function to the measurements made from event shape variables.

degrees of freedom corresponding to a confidence level of 0.34 with a fitted value of $\alpha_{\mathrm{s}}$ :

$\alpha_{\mathrm{s}}\left(m_{\mathrm{Z}}\right)=0.1215 \pm 0.0012 \pm 0.0061$.

The first error is experimental and the second error is theoretical. On the other hand, a fit with constant $\alpha_{\mathrm{s}}$ gives a $\chi^{2}$ of 65.1 for 12 degrees of freedom. The value of $\alpha_{\mathrm{s}}\left(m_{\mathrm{Z}}\right)$ thus obtained is in agreement with the value obtained in the power law ansatz analysis considering the experimental and the theoretical uncertainties.

Fig. 5b summarises the $\alpha_{\mathrm{s}}$ values determined by L3 from the $\tau$ lifetime measurement [30], Z lineshape [31] and event shape distributions at various 
energies, together with the QCD prediction obtained from a fit to the event shape measurements only. These measurements support the energy evolution of the strong coupling constant predicted by QCD.

The slope in the energy evolution of $\alpha_{\mathrm{s}}$ depends on the number of active flavours. We have performed a fit with $N_{f}$ as a free parameter along with $\alpha_{\mathrm{s}}$ and obtain the number of active flavours:

$N_{f}=5.0 \pm 1.3 \pm 2.0$,

where the first error is experimental and the second is due to theoretical uncertainties. The errors have been estimated by using the covariance matrix determined from experimental and overall errors on $\alpha_{\mathrm{s}}$ in the fit. This result agrees with the expectation $N_{f}=$ 5 .

\section{Summary}

We have measured distributions of event shape variables in hadronic events from $\mathrm{e}^{+} \mathrm{e}^{-}$annihilation at centre-of-mass energies from $30 \mathrm{GeV}$ to 189 $\mathrm{GeV}$. These distributions as well as the energy dependence of their first moments are well described by parton shower models.

The energy dependence of the first two moments has been compared with second order perturbative QCD with power law corrections for the non-perturbative effects. The fits of the five event shape variables agree with a universal power law behaviour giving $\alpha_{0}=0.537 \pm 0.070(\exp ) \pm 0.021$ (th). We find a non-negligible contribution from an $\mathscr{O}\left(\frac{1}{s}\right)$ term in describing the second moments of $1-T$, $B_{W}, B_{T}$ and $C$. Improvements in theoretical predictions for the second moments of jet broadening variables are clearly necessary.

The event shape distributions are compared to second order QCD calculations together with resummed leading and next-to-leading log terms. The data are well described by these calculations at all energies. The measurements demonstrate the running of $\alpha_{\mathrm{s}}$ as expected in QCD with a value of $\alpha_{\mathrm{s}}\left(m_{\mathrm{Z}}\right)$ $=0.1215 \pm 0.0012(\mathrm{exp}) \pm 0.0061(\mathrm{th})$. From the energy dependence of $\alpha_{\mathrm{s}}$, we determine the number of active flavours to be $N_{f}=5.0 \pm 1.3(\exp ) \pm 2.0$ (th).

\section{Acknowledgements}

We express our gratitude to the CERN accelerator divisions for the excellent performance of the LEP machine. We acknowledge with appreciation the effort of the engineers, technicians and support staff who have participated in the construction and maintenance of this experiment.

\section{References}

[1] L3 Collaboration, B. Adeva et al., Phys. Lett. B 284 (1992) 471; L3 Collaboration, O. Adriani et al., Phys. Rep. 236 (1993) 1.

[2] L3 Collaboration, M. Acciarri et al., Phys. Lett. B 411 (1997) 339.

[3] L3 Collaboration, B. Adeva et al., Nucl. Instr. Meth. A 289 (1990) 35; M. Chemarin et al., Nucl. Instr. Meth. A 349 (1994) 345; M. Acciarri et al., Nucl. Instr. Meth. A 351 (1994) 300; G. Basti et al., Nucl. Instr. Meth. A 374 (1996) 293; A. Adam et al., Nucl. Instr. Meth. A 383 (1996) 342.

[4] L3 Collaboration, M. Acciarri et al., Phys. Lett. B 371 (1996) 137.

[5] L3 Collaboration, M. Acciarri et al., Phys. Lett. B 404 (1997) 390; L3 Collaboration, M. Acciarri et al., Phys. Lett. B 444 (1998) 569

[6] Jetset 7.4 Monte Carlo Program: T. Sjöstrand, Comp. Phys. Comm. 82 (1994) 74.

[7] Herwig 5.9 Monte Carlo Program: G. Marchesini, B. Webber, Nucl. Phys. B 310 (1988) 461; I.G. Knowles, Nucl. Phys. B 310 (1988) 571; G. Marchesini et al., Comp. Phys. Comm. 67 (1992) 465.

[8] Ariadne 4.06 Monte Carlo Program: U. Pettersson, ARIADNE: A Monte Carlo for QCD Cascades in the Color Dipole Formulation, Lund Preprint, LU TP 88-5 (1988); L. Lönnblad, The Colour Dipole Cascade Model and the Ariadne Program, Lund Preprint, LU TP 91-11 (1991).

[9] Cojets 6.23 Monte Carlo Program: R. Odorico, Nucl. Phys. B 228 (1983) 381; R. Odorico, Comp. Phys. Comm. 32 (1984) 139; Erratum: 34 (1985) 43; R. Mazzanti, R. Odorico, Nucl. Phys. B 370 (1992) 23; R. Mazzanti, R. Odorico, Bologna preprint DFUB 92/1.

[10] L3 Collaboration, B. Adeva et al., Z. Phys. C 55 (1992) 39; S. Banerjee, M.Sc. Thesis, University of Mumbai, 1998.

[11] Yu.L. Dokshitzer, B.R. Webber, Phys. Lett. B 352 (1995) 451; B.R. Webber, hep-ph/-9510283; Yu.L. Dokshitzer et al., Nucl. Phys. B 511 (1997) 396; Yu.L. Dokshitzer et al., J. High Energy Physics 05 (1998) 3; Yu.L. Dokshitzer et al., Eur. Phys. J. direct C 3 (1999) 1; Yu.L. Dokshitzer, hep$\mathrm{ph} /-9911299$.

[12] DELPHI Collaboration, P. Abreu et al., Phys. Lett. B 456 (1999) 322. 
[13] Z. Kunszt, P. Nason, in Z Physics at LEP 1, CERN Report 89-08, vol. I., p. 373.

[14] S. Catani et al., Phys. Lett. B 263 (1991) 491; S. Catani et al., Phys. Lett. B 272 (1991) 368; S. Catani et al., Phys. Lett. B 295 (1992) 269; S. Catani et al., Nucl. Phys. B 407 (1993) 3; S. Catani et al., Phys. Lett. B 427 (1998) 377.

[15] Pythia 5.7 Monte Carlo Program: QCD parton shower and fragmentation process are taken from Jetset 7.4 [6]; T. Sjöstrand, CERN-TH-7112/93 (1993), revised august 1995; T. Sjöstrand, Comp. Phys. Comm. 82 (1994) 74.

[16] The L3 detector simulation is based on GEANT Version 3.15. See R. Brun et al., GEANT 3, CERN DD/EE/84-1 (Revised), September 1987. The GHEISHA program (H. Fesefeldt, RWTH Aachen Report PITHA 85/02 (1985)) is used to simulate hadronic interactions.

[17] R. Engel, Z. Phys. C 66 (1995) 203; R. Engel, J. Ranft, S. Roesler, Phys. Rev. D 52 (1995) 1459.

[18] S. Jadach, B.F.L. Ward, Z. Wạs, Comp. Phys. Comm. 79 (1994) 503.

[19] J.H. Field, Phys. Lett. B 323 (1994) 432; J.H. Field, T. Riemann, Comp. Phys. Comm. 94 (1996) 53.

[20] S. Jadach et al., Phys. Lett. B 390 (1997) 298.

[21] M. Skrzypek et al., Comp. Phys. Comm. 94 (1996) 216; M. Skrzypek et al., Phys. Lett. B 372 (1996) 289.
[22] Yu.L. Dokshitzer, Contribution to the Workshop on Jets at LEP and HERA (1990); N. Brown, W.J. Stirling, Rutherford Preprint RAL-91-049; S. Catani et al., Phys. Lett. B 269 (1991) 432; S. Bethke et al., Nucl. Phys. B 370 (1992) 310.

[23] S. Brandt et al., Phys. Lett. 12 (1964) 57; E. Fahri, Phys. Rev. Lett. 39 (1977) 1587.

[24] T. Chandramohan, L. Clavelli, Nucl. Phys. B 184 (1981) 365; MARK II Collaboration, A. Peterson et al., Phys. Rev. D 37 (1988) 1; TASSO Collaboration, W. Braunschweig et al., Z. Phys. C 45 (1989) 11.

[25] G. Parisi, Phys. Lett. B 74 (1978) 65; J.F. Donoghue, F.E. Low, S.Y. Pi, Phys. Rev. D 20 (1979) 2759.

[26] V. Blobel, DESY Report 84-118 (1984).

[27] S. Catani, M. Seymour, Phys. Lett. B 378 (1996) 287.

[28] Yu.L. Dokshitzer, B.R. Webber, Phys. Lett. B 404 (1997) 321; B.R. Webber, Nucl. Phys. Proc. Suppl. 71 (1999) 66; G. Salam, private communication.

[29] Particle Data Group, C. Caso et al., Eur. Phys. J. C 3 (1998) 1.

[30] L3 Collaboration, M. Acciarri et al., CERN-EP / 2000-040 (2000).

[31] L3 Collaboration, M. Acciarri et al., CERN-EP/2000-022 (2000). 